\title{
Periodic solutions to ZK-MEW equation in the form Lame equation
}

$$
\text { Chun-Huan Xiang }{ }^{1, a} \text {, Hong-Lei Wang }{ }^{2, b}
$$

\author{
${ }^{1}$ School of Public Health and Management, Chongqing Medical University, Chongqing, 400016, P.R. \\ China \\ ${ }^{2}$ College of medical informatics, Chongqing Medical University, Chongqing, 400016, P. R. China \\ aemail:w825900@163.com, bemail:w8259300@126.com
}

\begin{abstract}
The periodic solutions to ZK-MEW equation is investigated based on an auxiliary Lame equation and the perturbation method. The periodic solutions to ZK-MEW equation is given in the form of Jacobi functions and expressed by the hyperbolic functions, the trigonometric functions with different modulus $m$. The results are simply discussed. This method is more powerful to seek the exact solutions of the nonlinear partial differential equations in mathematical physics.
\end{abstract}

Keywords: nonlinear, ZK-MEW equation, Lame equation, evolution equation

\section{Introduction}

The investigation about exact solutions of nonlinear equations is an important subject because they play important role in understanding the nonlinear problems. Recently, many authors presented various powerful method to deal with this interest subject, such as Backlund transformation [1], Darboux transformation [2], the extended tanh-function method [3], the F-expansion method [4], projective Riccati equations method[5], the Jacobian elliptic functions expansion method[6] and so on [7-10]. Recently , vast research results [11-15] have been obtained in this field. The aim of this paper is to extend the Lame equation method to solve the nonlinear differential equations ZK-MEW equation [16].

\section{The nonlinear ZK-MEW equation and Lame equation method}

The ZK-MEW equation governs the behavior of weakly nonlinear ion-acoustic waves in a plasma comprising cold ions and hot isothermal electrons in the presence of a uniform magnetic field [17]. The nonlinear ZK-MEW equation is given in the form as follow

$$
\left(u_{t}+a\left(u^{3}\right)_{x}+\left(b u_{x t}+r u_{y y}\right)_{x}=0\right.
$$

where $a, b$ and $r$ are known constants. Using the wave variable $\xi=x+y-c t$ and proceeding as before we find

$$
c u^{\prime}+a\left(u^{3}\right)^{\prime}-b c u^{\prime \prime \prime}+r u^{\prime \prime \prime}=0
$$

Integrating (2) with respect to $\xi$ and neglecting constants of integration we obtain

$$
-c u+a u^{3}+(r-b c) u^{\prime \prime}=0
$$

Lame equation with a kernel function expressed in terms of $d n(\xi)$ reads

$$
\frac{d^{2} \Lambda_{n}^{(p)}(\xi)}{d \xi^{2}}+\left[p(p+1) \mathrm{dn}^{2}(\xi)-B_{n}^{(p)}\right] \Lambda_{n}^{(p)}(\xi)=0, \quad p=1,2, \ldots, \quad n=1,2, \ldots, 2 p+1 .
$$

Here $\Lambda_{n}^{p}(\xi)$ is the $n$th eigenfunction of Lame Eq. (4), corresponding to the eigenvalue $B_{n}^{p}(\xi)$

For Jacobi elliptic functions, we have

$\mathrm{cn}^{2} \xi=1-\mathrm{sn}^{2} \xi, \quad \mathrm{dn}^{2} \xi=1-m^{2} \mathrm{sn}^{2} \xi$, and $\frac{d}{d \xi} \operatorname{sn} \xi=\mathrm{cn} \xi \operatorname{dn} \xi, \quad \frac{d}{d \xi} \mathrm{cn} \xi=-\operatorname{sn} \xi \operatorname{dn} \xi, \quad \frac{d}{d \xi} \operatorname{dn} \xi=-m^{2} \operatorname{sn} \xi \operatorname{cn} \xi$.

$s n(\xi) c n(\xi) d n(\xi)$ are Jacobi elliptic sine function, cosine function and Jacobi elliptic function of the third kind with the modulus $m(0<m<1)$, respectively. When $m \rightarrow 0$ or $m \rightarrow 1$, Jacobi elliptic functions asymptotically go into trigonometric or hyperbolic one: 


$$
\begin{aligned}
& \operatorname{sn} \xi \rightarrow \sin \xi, \quad \text { cn } \xi \rightarrow \cos \xi, \quad \operatorname{dn} \xi \rightarrow 1, \quad \text { when } m \rightarrow 0 ; \\
& \operatorname{sn} \xi \rightarrow \tanh \xi, \quad \text { cn } \xi \rightarrow \operatorname{sech} \xi, \quad \operatorname{dn} \xi \rightarrow \operatorname{sech} \xi, \quad \text { when } m \rightarrow 1 .
\end{aligned}
$$

For a given nonlinear equation as the follow

$$
G\left(u, u_{x}, u_{t}, u_{x x}, u_{t t}, u_{x t}, \ldots\right)=0
$$

Where $u_{x}=\partial u / \partial x, u_{x x}=\partial^{2} u / \partial x^{2}, u_{x t}=\partial^{2} u / \partial x \partial t, \ldots$ with the expression as $\xi=x+y-c t$, where $c$ denotes the wave speed, then leads to an ordinary differential equation.

$$
G\left(u, u_{\xi}, u_{\xi \xi}, u_{\xi \xi \xi}, \ldots\right)=0
$$

The minimal expand is used to obtain the approximation evolution solution for nonlinear equation.

$$
u(\xi)=u_{0}(\xi)+p u_{1}(\xi)+p^{2} u_{2}(\xi)+\ldots
$$

Where $p$ is small parameter, $u_{0}(\xi), u_{1}(\xi), u_{2}(\xi)$ represent the zeroth-order, first-order and secondorder solution, respectively.

Substituting Eq. (8) into Eq.(7) and equating to zero the coefficients of all powers of $p$ yields a set of the second-order differential equations in terms of $u_{0}(\xi), u_{1}(\xi), u_{2}(\xi)$, which called the zeroth-order, first-order, second-order, ... . equations, respectively. All equations can be solved by using auxiliary Lame equation method.

\section{Numerical example}

We employ the Lame equation method for the nonlinear equations (3), the useful expression (8) is used. Substituting Eq. (8) into Eq. (3), and equating the coefficients of all powers of $p$ to zero, the first three equations as follows:

$$
\begin{gathered}
a u_{0}^{3}-c u_{0}-b c u_{0}^{\prime \prime}+r u_{0}^{\prime \prime}=0 \\
3 a u_{0}^{2} u_{1}-c u_{1}-b c u_{1}^{\prime \prime}+r u_{1}^{\prime \prime}=0 \\
3 a u_{0} u_{1}^{2}+3 a u_{0}^{2} u_{2}-c u_{2}-b c u_{2}^{\prime \prime}+r u_{2}^{\prime \prime}=0
\end{gathered}
$$

Eq.(9) can be solved by using auxiliary elliptic equation method and suppose.

$$
u_{0}=a_{0}+a_{1} d n(\xi)+a_{2} d n(\xi)^{2}
$$

Submitting the equation (12) into Eq.(9), the parameter $a_{0}, a_{1}, a_{2}$ can be easily determined

$$
u_{0}=\frac{(b c-r)\left(m^{2}-2\right)}{c} d n(\xi)
$$

Putting Eq. (13) into Eq.(10) yields

$$
u_{1}^{\prime \prime}+\left(\frac{3 a(r-b c)\left(m^{2}-2\right)^{2}}{c^{2}} d n(\xi)^{2}-c\right) u_{1}=0,
$$

By employing Eq. (5) and (6), we can obtain the solution as the follow

$$
u_{1}=\operatorname{Bsn}(\xi) c n(\xi) d n(\xi)
$$

Submitting the equation (13) and (15) into Eq. (11), we obtain

$$
\frac{3 a\left(2-m^{2}\right) d n(\xi)}{c}(B s n(\xi) c n(\xi) d n(\xi))^{2}+\left(\frac{3 a\left(2-m^{2}\right)^{2} d n(\xi)^{2}}{c^{2}}-\frac{c}{r-b c}\right) u_{2}+u_{2}^{\prime \prime}=0
$$

The solution of Eq.(16) is supposed as

$$
u_{2}=n_{0}+n_{1} d n(\xi)^{2}+n_{2} d n(\xi)^{4}
$$

Submitting the equation (17) into Eq.(16) and equating the coefficient of $d n(\xi)$ to zero, we obtain the following equation:

$$
-2 n_{1}+2 m^{2} n_{1}-\frac{c n_{0}}{r-b c}=0
$$




$$
\begin{aligned}
& \frac{12 a-12 a m^{2}+3 a m^{4}}{c^{2}} n_{0}+\left(8-4 m^{2}-\frac{c}{r-b c}\right) n_{1}+12\left(m^{2}-1\right) n_{2}=0 \\
& \frac{12 a-12 a m^{2}+3 a m^{4}-6 c^{2}}{c^{2}} n_{1}+\left(32-16 m^{2}-\frac{c}{r-b c}\right) n_{2}=0
\end{aligned}
$$

From the above equations, we obtain the solution as

$$
\begin{gathered}
n_{1}=\frac{c n_{0}}{2(r-b c)\left(m^{2}-1\right)} \\
n_{2}=\frac{2\left(12 a-12 a m^{2}+3 a m^{4}\right)(r-b c)^{2}\left(m^{2}-1\right)+\left(\left(8-4 m^{2}\right)(r-b c)-c\right) c^{3}}{-24 c^{2}(r-b c)^{2}\left(m^{2}-1\right)^{2}} n_{0}
\end{gathered}
$$

where $n_{0}$ is a nonzero constant parameter.

The Eq. (17) is read as

$u_{2}=n_{0}+\frac{c n_{0}}{2(r-b c)\left(m^{2}-1\right)} d n(\xi)^{2}+\frac{2\left(12 a-12 a m^{2}+3 a m^{4}\right)(r-b c)^{2}\left(m^{2}-1\right)+\left(\left(8-4 m^{2}\right)(r-b c)-c\right) c^{3}}{-24 c^{2}(r-b c)^{2}\left(m^{2}-1\right)^{2}} n_{0} d n(\xi)^{4}$

Submitting Eq. (13) (15) and (18) into Eq.(8), we obtain the evolution solutions for Eq.(3) as the follow

$$
\begin{aligned}
& u(\xi)=\frac{(b c-r)\left(m^{2}-2\right)}{c} d n(\xi)+p B s n(\xi) c n(\xi) d n(\xi)+n_{0}+\frac{c n_{0}}{2(r-b c)\left(m^{2}-1\right)} d n(\xi)^{2}+ \\
& \frac{2\left(12 a-12 a m^{2}+3 a m^{4}\right)(r-b c)^{2}\left(m^{2}-1\right)+\left(\left(8-4 m^{2}\right)(r-b c)-c\right) c^{3}}{-24 c^{2}(r-b c)^{2}\left(m^{2}-1\right)^{2}} n_{0} d n(\xi)
\end{aligned}
$$

The solution for nonlinear ZK-MEW equation in the form Eq.(1) is written as

$$
\begin{aligned}
& u(x, y, t)=\frac{(b c-r)\left(m^{2}-2\right)}{c} d n(x+y-c t, m)+p B s n(x+y-c t, m) c n(x+y-c t, m) d n(x+y-c t, m) \\
& +\frac{2\left(12 a-12 a m^{2}+3 a m^{4}\right)(r-b c)^{2}\left(m^{2}-1\right)+\left(\left(8-4 m^{2}\right)(r-b c)-c\right) c^{3}}{-24 c^{2}(r-b c)^{2}\left(m^{2}-1\right)^{2}} n_{0} d n(x+y-c t, m)^{4} \\
& +n_{0}+\frac{c n_{0}}{2(r-b c)\left(m^{2}-1\right)} d n(x+y-c t, m)^{2}
\end{aligned}
$$

When $m \rightarrow 0$ or $m \rightarrow 1$, the Eq. (19) is reduced into trigonometric or hyperbolic solutions for Eq. (3)

The simulation figure for Eq.(19) is shown in Fig. 1, the parameters are $b=0.1 ; c=5 ; m=0.2 ; r=0.25$; $p=0.001 ; B=2 ; n_{0}=0.4 ; t=0.1 ; a=0.12 ; x \in(0,5), \quad y \in(0,5)$, respectively.

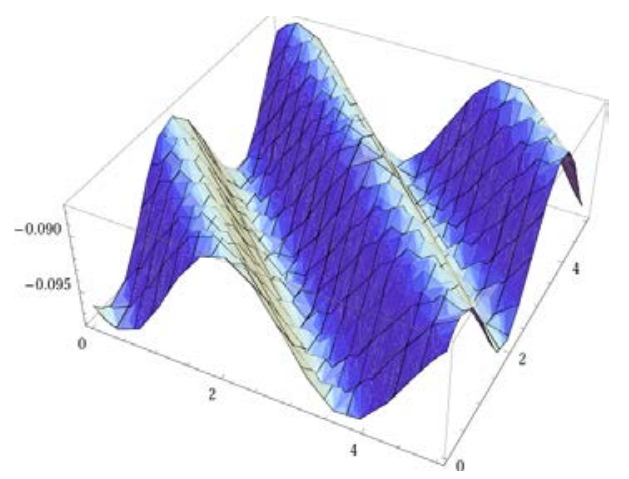

Fig.1 The simulation result is shown for Eq. (19)

\section{Conclusions}

The nonlinear ZK-MEW equation is investigated in this manuscript. The Lame equation method was successfully used to establish travelling wave solutions, which performance is reliable and effective. 
Many well known nonlinear wave equations were handled by this method. The simulation figures for the evolution solutions are shown. We believe that this method should play an important role for finding exact solutions in the mathematical physics.

\section{References}

[1] D.C.Lu, B.J.Hong, Backlund transformation and n-soliton-like solutions to the combined KdVBurgers equation with variable coefficients.Int.J.Nonlinear Sci. 1(2):3-10 (2006).

[2] V.A. Matveev, M.A. Salle, Darboux transformations and solitons. Berlin, Heidelberg: Springer-Verlag. (1991).

[3] E. G. Fan, Extended tanh-function method and its applications to nonlinear equations. Phys. Lett. A .277:212-220 (2000).

[4] Y. B. Zhou, M. L. Wang, Y. M. Wang, Periodic wave solutions to a coupled KdV equations with variable coefficients. Phys. Lett. A. 308:31-37 (2003).

[5] D.C.Lu,B.J.Hong, New exact solutions for the (2+1)-dimensional Generalized Broer-Kaup System. Appl. Math. Comp. 199(2):572-580 (2008).

[6] S. S. Liu, Z. T. Fu , S. D. Liu, Q. Zhao, Jacobi elliptic function expansion method and periodic wave solutions of nonlinear wave equations. Phys. Lett. A. 289(1-2):69-74 (2001).

[7] Y. R. Shi , P. Guo, K. P. L,W. S. Duan, Expansion method for modified Jacobi elliptic function and its application. Acta. Phys . Sin. 53(3):3265-3270 (2004)(in Chinese)

[8] J .G.Wu, M. Zhang, L. M. Shi,W. L. Zhang etal, The extended expansion method for Jacobi elliptic function and new exact periodic solutions of Zakharov equations. Acta. Phys. Sin. 56(9):5054-5060 (2007)(in Chinese)

[9] R. Conte, M. Musette, Link between solitary waves and projective Riccati equations. Phys. A: Math. Gen. 25(21):5609-5623 (1992).

[10] Y. R. Shi, K. P. L,W. S. Duan etal, Explicit and exact solutions of the combined KdV equation. Acta. Phys.Sin. 52(2):267-271(2003)(in Chinese).

[11] H. Zhang, New extract travilling wave solution for some nonlinear evoluation equations . Chaos, Soliton \& Fractals. 26:921 (2005).

[12] D.Wang , H. Zhang, Farther improved F-expansion method and new extract solution of Konopeknenko - Dubrovsky equation. Chaos , Soliton \& Fractals ,25:601-610 (2005).

[13] M.A.Abdou , Generalized solitonary and periodic solutions for nonlinear partial differential equations by the Exp-function method. Nonlinear Dyn.DOI. 10.1007/ s11071 - 007 - 9250-1.

[14] M.A.Abdou, on the variational iteration method. Phys. Lett. A. 366:61-68 (2007).

[15] S.A.Khuri, Travelling wave solution for nonlinear differential equations: Aunified ansatz approach. Chaos , Soliton \& Fractals. 32:252-258 (2007).

[16] M. Inc, New exact solutions for the ZK-MEW equation by using symbolic computation. Appl. Math. Comput. 189:508 (2007).

[17] A.-M. Wazwaz, Exact solutions with solitons and periodic structures for the Zakharov-Kuznetsov equation and its modified form, Communications in Nonlinear Science and Numerical Simulation, 10, 597-606, 2005. 